als theurer erweisen, wenn nicht der erste Anschaffungspreis, sondern die wirkliche Leistung in Betracht gezogen wird, wie ich es schon oben bei Barbier's System nachgewiesen habe. Auch möge man in Bezug auf die Kosten nicht übersehen, was an Bodenfläche, Gebäuden, Fundamenten und Pumpen erspart wird.

Einen Nachtheil haben allerdings die Plattenthürme gegenüber den Koksthürmen, der übrigens bei ihrerVerwendung zurVerringerung des Bleikammerraumes gar nicht in Betracht kommt, nämlich den, dass sie im Verhältniss zu den mächtigen Koksthürmen nur wenig Flüssigkeit und Gas enthalten, und mithin die unvermeidlichen Verschiedenheiten im Betriebe sich nicht so gut ausgleichen. Das habe ich nie geleugnet; aber es lässt sich fast immer durch Combination des Plattenthurms mit einigen als Regulatoren dienenden Thonflaschen oder Steinkästen verbessern. In anderen Fällen, z. B. bei Gay-Lussacthürmen und Gloverthürmen, wird man einem Plattenthurme den Haupttheil der Aufgabe zuweisen können, während beim Gay-Lussac ein gewöhnlicher Koksthurm die abgekühlten armen Gase, beim Glover ein Steinthurm die allzu heissen und mit Flugstaub verunreinigten ersten Gase aufnimmt. In beiden Fällen bat man den Vortheil, dass kei der geringen Höhe des Plattenthurmes dieser Apparat unter bez. auf den anderen Thurm gesetzt und durch ein einziges Pumpen der Säure für beide zusammen bedient wird. In dem oben erwähnten Falle in Amerika, wo ein Plattenthurm ohne Koksthurm vollkommen befriedigend als Gay-Lussacthurm functionirt, ist er so aufgestellt, dass die Nitrose ohne Pumpen direct auf den Gloverthurm fliesst, was ja bei einem Koksthurme kaum durchführbar wäre.

Züricb, Mai 1893.

\section{Eine Modification \\ der Neubauer'schen Caramelbestimmung in mit Zucker gebranntem Kaffee.}

(Mittbeilung aus dem Laboratorium des GeriçhtsChemikers Dr. Bein-Berlin.)

\section{Von}

\section{Dr. J. Stern und Dr. A. Prager.}

Die zuerst von Liebig (Chem. Centr. 1866, 575) empfohlene Methode des Kaffeebrennens mit Zucker zum Zwecke der dauernden Erhaltung der beim Brennen sich bildenden, das Aroma des Kaffees darstellenden aromatischen Principe, hat sich in den letzten Jahren in grösserem Maassstabe bei uns eingebürgert und wird von bedeutenden Firmen nicht zum Nachtheil des consumirenden Publikums ausgeführt. Die auf diese Art gebrannten, sich z. Th. einer grossen Beliebtheit erfreuenden Kaffees sind insofern Gegenstand häufiger Untersuchungen, als durch einen übermässigen Zusatz von Zucker beim Rösten der Caramelgehalt eine solche Höhe erreichen kann, dass durch den relativ niedrigen Kaffeegehalt der Nutzen dieser Art des Röstens aufgehoben werden kann. Es ist daher von grosser Wichtigkeit, Kaffees bei Händlern und Producenten auf ihren Caramelgehalt controliren zu können, um zu ermessen, ob es sich bei derartigen Kaffeesorten um eine in gewinnsüchtiger Absicht beigebrachte Gewichtsvermehrung handelt.

Die einzige Methode der Caramelbestimmung in mit Zucker gebrannten Kaffees, welche zuverlässige Resultate gibt, ist die Neubauer'sche. Nach derselben werden abgewogene Mengen des fraglichen mit Zucker gebrannten, nicht zerkleinerten Kaffees mit Äther gleichmässig befeuchtet, mit gewogenen Mengen kochenden Wassers übergossen und unter fleissigem Umrühren $1 / 4$ Stunde damit in Berührung gelassen. Nach dieser Zeit wird die Flüssigkeit abfiltrirt und auf ein bestimmtes Volumen gebracht. Der Extract wird durch Abdampfen eines Theils der Lösung und durch Trocknen im Luftstrom quantitativ bestimmt.

Da bei dieser Behandlung ein jeder selbst nicht mit Zucker gebrannter - Kaffee je nach dem stärkeren oder geringeren Grade der Röstung wechselnde Mengen von Extract abgibt, so wurden dieselben Bestimmungen auf verschieden gebrannte Kaffeesorten ausgedehnt und der Extractgehalt bestimmt.

Neubauer fand im Mittel für verschiedene ungleich stark ohne Zucker gebrannte Kaffees einen Extractgehalt von 1,23 Proc. - Die nach dieser Methode von uns ausgeführten Extractbestimmungen in vielen verschiedenen, ohne Zucker gebrannten Kaffees ergaben einen Durchschnitt von 1,29 Proc. und wir haben diese Zahl unseren Versuchen zu Grunde gelegt.

Unsere Modification der Neubauer'schen Methode, welche auszuarbeiten wir wegen der in grosser Anzahl zugleich auszuführenden Bestimmungen veranlasst wurden, hat den Vortheil, dass das zeitraubende und viel Gefässe erfordernde Eindampfen und Trocknen bei bestimmter Temperatur in Wegfall 
kommt. Das gleiche Ziel erreichen wir dadurch, dass wir aus dem specifischen Gewichte der hergestellten Extractlösung auf deren Caramelgehalt schliessen. vielen nach dieser Methode untersuchten Kaffeesorten mögen folgende angeführt sein, von denen einzelne mit hohem Caramelgehalte beanstandet wurden:

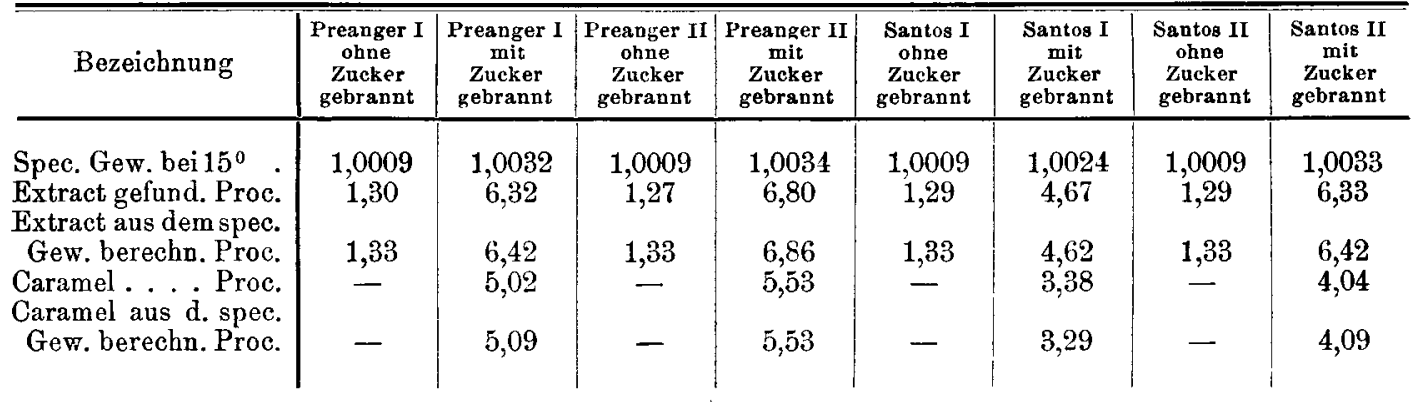

Um einen sicheren Rückschluss machen zu können, baben wir aus reinem von uns selbst dargestellten Caramel Lösungen von bekanntem Gehalt bereitet und deren specifische Gewichte mittels Pyknometer ermittelt. Sie sind in folgender Tabelle zusammengestellt:

\begin{tabular}{|c|c|c|}
\hline \multicolumn{2}{|c|}{ Caramelgehalt der Lòsung } & Spec. Gew. bei $15^{\circ}$ \\
\hline 0,1 & Proc. & 1,00066 \\
\hline 0,2 & - & 1,00104 \\
\hline 0,3 & - & 1,00142 \\
\hline 0,4 & - & 1,00180 \\
\hline 0,5 & - & 1,00220 \\
\hline 0,6 & - & 1,00256 \\
\hline 0,7 & - & 1,00292 \\
\hline 0,8 & - & 1,00330 \\
\hline 0,9 & - & 1,00368 \\
\hline 1,0 & - & 1,00406 \\
\hline
\end{tabular}

Bei Anwendung dieser Tabelle ist von der Annahme ausgegangen worden, dass das specifische Gewicht der Lösungen des $\mathrm{Ge}^{-}$ sammtextractes (also des Caramels + den sonstigen Extractivstoffen) von mit Zucker gebrannten Kaffees nicht wesentlich durch den - im vorliegenden Falle etwa $1 / 3$ betragenden - Gehalt an Extractivstoffen des Kaffees selbst beeinflusst wird. Es wird demgemäss kein für die Praxis in's Gewicht fallendes Moment sein, wenn die Lösung als reine Caramellösung betrachtet wird.

Die Berechnung stellt sich folgendermaassen:

Bei Anwendung von $30 \mathrm{~g}$ Kaffee, deren Extract auf 250 ce gebracht wird, erhält man Lösungen, deren specifische Gewichte je nach der Höhe des Extractgehaltes zwischen 1,0009 und 1,00368 liegen. Diesen Lösungen entspricht nach $\mathrm{Neubauer}$ ein Extractgehalt im Kaffee von 1,33 bis 7,5 Proc. oder ein Procentgehalt der Lösung selbst von 0,16 bis 0,9 Proc. Wir bestimmen also aus dem specifischen Gewichte der Lösung den Gehalt derselben an Extract (als reines Caramel) aus der Tabelle und finden durch eine einfache Rechnung den Extract- bez. Caramelgehalt des Kaffees selbst. Unter den

\section{Apparate.}

Trockenverfahren von Büttner und Meyer (D.R.P. No. 67022). Den eintretenden heissen Feuergasen soll zunächst ein Theil der Wärme durch Strahlung entzogen werden, bevor sie das Trockengut selbst treffen. Daher ist der Eintritt der heissen Gase $A$ (Fig. 150 bis 152) so hoch

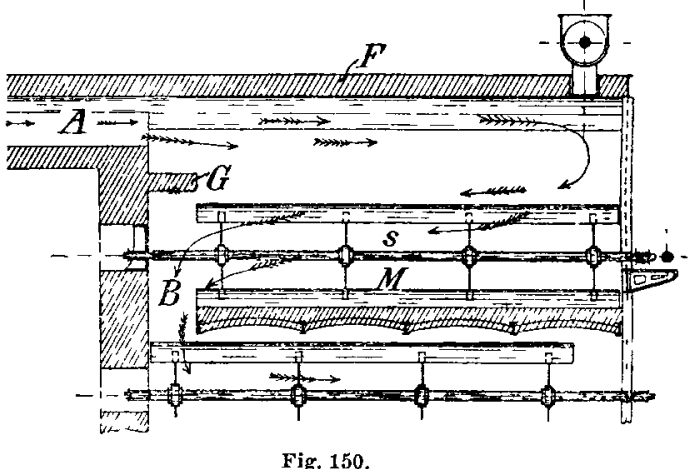

und in solcher Richtung angelegt, dass ein Stoss der Gase auf das Trockengut, welches, in den Mulden $M$ liegend, durch die Schaufelräder $s$ stetig gewendet wird, vermieden wird. Oberhalb der Schaufelräder befindet sich ein durch ein feuerfestes Gewölbe $F$ begrenzter Raum von genügender Höhe. Die Gase theilen ihre Wärme dem Gewölbe und dieses durch Strahlung dem kalten und nassen zu trocknenden Material mit, dessen Temperatur gleichwobl wegen der eintretenden Verdunstung unter $100^{\circ}$ bleibt. Abgekühlt und mit den in der obersten Etage erzeugten Wasserdämpfen vermischt, treten die Gase alsdann durch Kanal $B$ in die darunter liegende Etage über, wo sie nunmehr obne Schaden durch directe Berührung wirken können.

Der Weg der Gase durch die oberste Etage kann entweder (nach Fig. 150 u. 151) so angeordnet werden, dass sie unter dem 
Gewölbe hin und durch den Wenderaum zurückgeführt werden, um dicht unterhalb ihrer Eintrittsstelle in den Kanal $B$ zu fallen, oder (nach Fig.152) so, dass sie nur

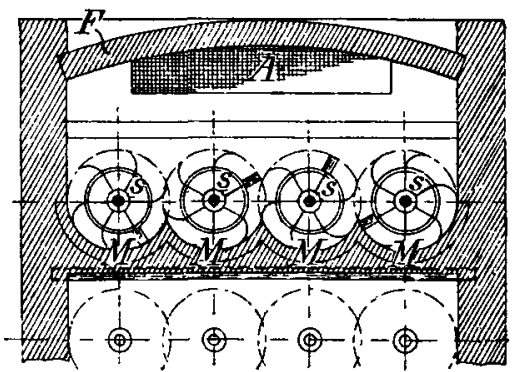

Fig. 151.

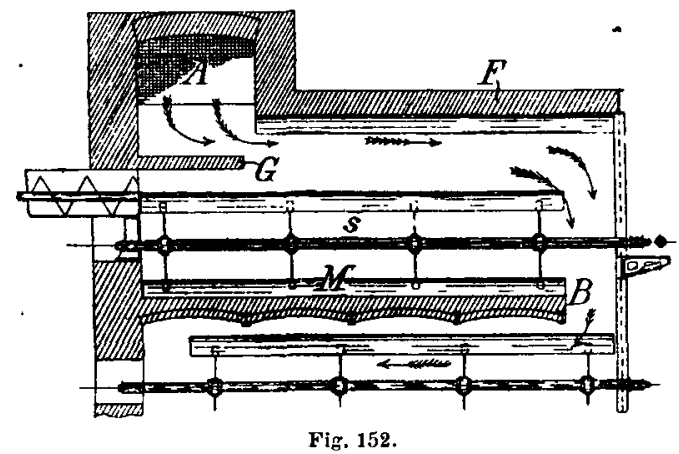

über die Wender hinstreichen, um auf der dem Eintritt entgegengesetzten Seite unten abgeführt zu werden. Im letzteren Falle ist die Wirkung auf das Trockengut in dieser Etage weniger stark.

Vacuumtrockenapparat. L. Storch (Ber. österr. G. 1893 S. 13) verwendet ein $5 \mathrm{~cm}$ weites, beiderseits verengtes Glasrohr $B$ (Fig. 153), um durch gute Korkstopfen

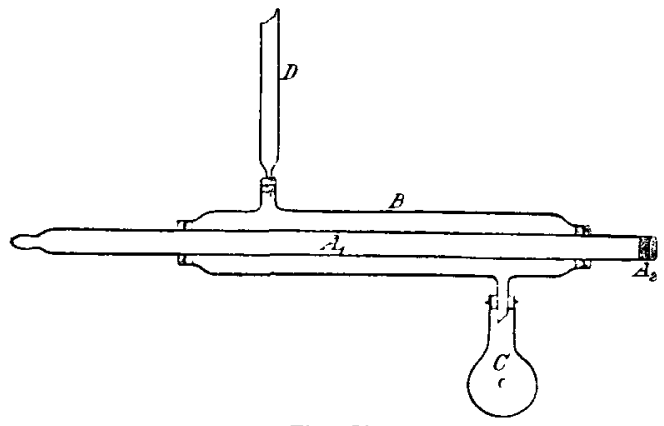

Fig. 153.

fixirt, das Rohr $A$ aufzunehmen. Der in den Kolben $C$, der vortheilhaft aus Kupfer hergestellt wird und etwa 50 cc fasst, reichende Ansatztheil ist schief abgeschliffen. Der andere, nach aufwärts gerichtete Ansatz trägt das etwa $1 \mathrm{~m}$ lange Kühlrohr $D$ von etwa $2 \mathrm{~cm}$ Durchmesser. $A$ hat eine Weite von $2 \mathrm{~cm}$ und wird um 12 bis $15 \mathrm{~cm}$ länger als $B$ gewählt, so dass ein etwa $10 \mathrm{~cm}$ langes Stück aus dem Heizrohr herausreicht. Das Rohr $B$ wird durch eine Kühlerklammer gehalten, $C$ ruht auf einem Eisendrahtdreieck, wird mit 5 bis 10 cc Heizflüssigkeit beschickt und mit einer kleinen Flamme erbitzt. Man gibt dem Apparat eine schwache Neigung gegen den Kolben $C$.

Als Heizflüssigkeiten benutzt man Aceton, Methylalkohol, Benzol, Toluol, Amylalkohol und höher siedendes $X y l o l$, so dass mit dem Apparate Temperaturen von 56 bis $142^{0} \mathrm{zu}$ erreichen sind. Bei Verwendung noch niedriger siedender Flüssigkeiten wie Aceton reicht das Kühlrohr nicht aus; man müsste einen Kühler anwenden, wodurch der Apparat an Handlichkeit einbüssen würde. Innerhalb weniger Minuten ist die Anheizung vollendet; der Innenraum von $\boldsymbol{A}$ zeigt die Temperatur der siedenden Flüssigkeit. Trägt man Sorge, dass die Dämpfe bis in eine Höhe von 10 bis $20 \mathrm{~cm}$ in dem Rohr $D$ aufsteigen, so ist man von geringen Druckschwankungen in der Gasleitung unabhängig. Verf. hat den Apparat oft 10 Stunden im Gange gehabt, ohne eine Regelung anbringen $\mathrm{zu}$ müssen.

Die zu erhitzende Probe wird in Schiffchen in das Innere von $A$ eingeführt. Handelt es sich um Wasserbestimmungen, so bringt man in den Theil $A_{1}$ eine aus einem Stück einer breiten Eprouvette geschnittene Röhre, die entsprechend mit einem Drahtstück als Handhabe zum Einführen und Herausziehen armirt und mit Chlorcalcium zwischen Wattepfropfen versehen ist. Dann verschliesst man $A_{2}$ mit einem Gummistopfen mit einer Bohrung, der ein aus einem engen Glasrohr hergestelltes Quecksilbermanometer trägt. Man saugt die Luft aus dem Apparate, schliesst den Saugschlauch durch einen Schraubenquetschhahn und ersieht an dem Manometer, inwieweit der Apparat Vacuum hält.

Gasofen für metallurgische Versuche. Nach W. Borchers (Stahleisen, gef. eing. Sonderabdr.) ruht der Ofen $V$ (Fig. 154 und 155) auf der gusseisernen Platte $p$; die mit gusseisernen Mänteln $m$ versehenen Wärmespeicher $W$ sind mit Scharmottekugeln gefüllt, welche auf Rosten $v^{*}$ ruhen.

Man schliesst die Öffnung $Z$ des Speichers $W^{1}$, die Öffnung $F$ des Speichers $W$, wodurch gleichzeitig die öffnungen $Z$ in $W$ und $F$ in $W^{1}$ geöffnet werden. Nun lässt man aus Rohr $R$ Gas in den Ofen treten, entzündet dasselbe und schliesst dann die Öffnung des Heizraumes in der 
Vorderwand. Die Flamme wird dann im Verbrennungsraume von links nach rechts ziehen, die Verbrennungsgase gehen durch $W^{1}$ in den Schornstein. Sobald die Wandungen im Verbrennungsraume zu glühen anfangen, öffoet man den Gashahn des Rohres $R^{1}$, schliesst das Rohr $R$, dann die Öffnung $Z$ in $W$ und möglichst gleichzeitig die Öffnung $F$ in $W^{\mathrm{i}}$. Die zur Verbrennung erforderliche Luft wird sich, während sie in $W^{1}$ aufsteigt, schon etwas anwärmen.
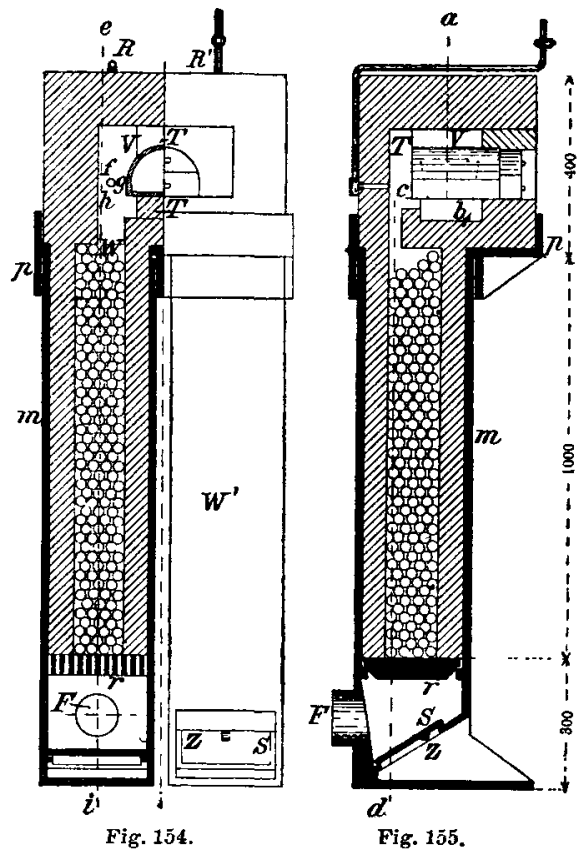

Die Flamme nimmt jetzt ihren Weg von rechts nach links. Die Verbrennungsgase wärmen auf ihrem Wege zum Schornstein die Steinmassen des Speichers $W$ an. Nach 30 bis 60 Minuten offnet man wieder das Rohr $R$, schliesst $R^{1}, Z$ in $W^{1}$ und $F$ in $W$. Die Flamme hat dann wieder die ursprüngliche Richtung u. s. f.

Thermom et er von F. v. L u p in (D.R.P. No. 66445). An mit Alkohol gefüllten Minimumthermometern macht sich häufig der Übelstand bemerkbar, dass vom Fadenende Flüssigkeit abdunstet und sich am Rohrende condensirt, sodass die Angaben des Thermometers unrichtig werden. Dies lässt sich vermeiden, wenn man Schwefelsäure von 36 bis 40 Proc. Monohydratgehalt oder Chlorcalciumlösung mit 15 Proc. Gehalt zur Füllung benutzt. Diese Flüssigkeiten lassen bei den in Betracht kommenden Temperaturen kein Wasser abdunsten und gefrieren erst unter $80^{\circ}$.

Messapparat für höhere Temperaturen von L. Damaze (D.R.P. No.67331) beruht auf der angeblichen Thatsache, dass die durch Temperaturänderungen bewirkte Ausdehnung des Glimmers der Zusammenziehung des Steinguts gleich ist und umgekehrt, sobald nur gewisse Temperaturgrenzen nicht überschritten werden und die beiden Körper eine bestimmte Länge haben. Eine derartig aus einzelnen cylindrischen Stücken zusammengesetzte Säule wird in ein Metallrohr gebracht, das der zu messenden Temperatur ausgesetzt wird. Hierbei ändert sich die Länge der umschlossenen Säule nicht, während das Metallrohr bei steigender oder sinkender Temperatur sich verlängert bez. verkürzt. Da nun Rohr und Säule an dem einen Ende mit einander verbunden sind, findet an ihrem anderen Ende eine Verschiebung statt, die in geeigneter Weise auf einen Zeiger übertragen wird.

Zur optischen Bestimmung hoher Temperaturen wird nach $H$. Le Chatelier (C. r. 114 S. 214, 340 und 391) von der gesammten Strahlung durch ein rothes Glas ein beschränkter Wellenlängenbereich ausgesondert und mit einer kleinen Erdöllampe verglichen.

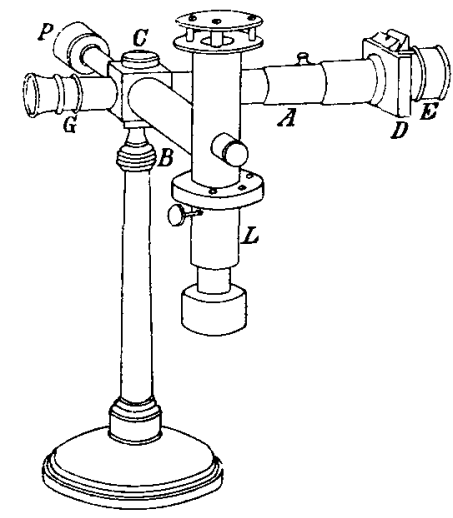

Fig. 156.

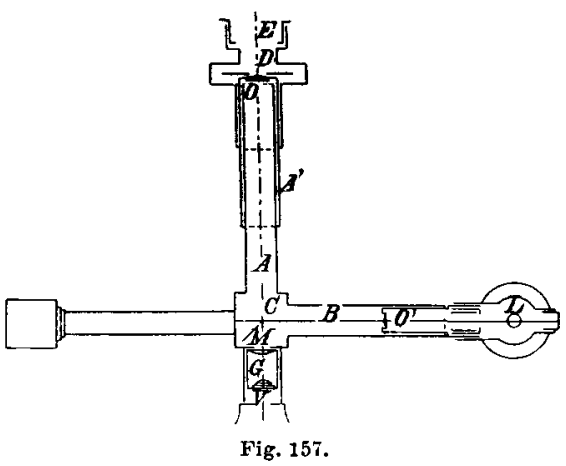

Die Erdöllampe $L$ (Fig. 156 u. 157) ist mit dem Photometer von Cornu vereinigt, bei welchem das Mikroskop durch Ocular $G$ ersetzt ist. Der Lichtschein der Lampe trifft 
durch Linse $O_{1}$ auf den, unter einem Winkel von $45^{\circ}$ in $C^{\prime}$ befestigten Spiegel $M$, während die andere Hälfte des Gesichtsfeldes durch das Bild des zu untersuchenden Lichtscheines ausgefüllt wird. Beide Lichtbilder werden gleichgemacht durch die stellbare Öffnung in Platte $D$ oder rothe Gläser $E$. Um aus der so gemessenen Lichtmenge die Temperatur berechnen zu können, legte Chatelier die Schmelzpunkte nach Violle (Gold $=1045$, Palladium $=1500$, Platin $=1775$ ) zu Grunde.

Zu Messungen des Emissionsvermögens benutzte er kleine Kugeln oder kleine Cylinder ron $5 \mathrm{~mm}$ Durchmesser, in welche längs eines Durchmessers ein Loch von $1 \mathrm{~mm}$ Durchmesser und $4 \mathrm{~mm}$ Tiefe gebohrt war; für rothe Strahlen bei ungefähr $1300^{\circ}$ erhielt er:

\begin{tabular}{lc}
\multicolumn{1}{c}{ Körper } & Emissionsvermögen \\
$\mathrm{Fe}_{3} \mathrm{O}_{4}$ & $\mathbf{1}$ \\
$\mathrm{Pd}$ & 0,6 \\
$\mathrm{Pt}$ matt & 0,4 \\
$\mathrm{Pt}$ polirt, weisser Thon & 0,25 \\
$\mathrm{MgO}$ & 0,1
\end{tabular}

Für rotbglühendes Eisen, welches an seiner Oberfläche stets oxydirt ist, darf man somit das Emissionsvermögen als der Einheit gleich ansehen. Die Intensität der Strablung ist deshalb unabhängig von der Temperatur der Umgebung und von dem Glanze der Oberfläche.

Versuche mit Platin bei verschiedenen Temperaturen ergaben:

Strahlung
0,00031
0,0011
0,0032
0,0043
0,011
0,073
0,205
0,210
0,480
0,60
1,40
1,90
2,80

Temperature
$730^{\circ}$
800
855
880
950
1080
1180
1200
1290
1300
1420
1450
1500
1775

Schmelzend. Platin $\mathbf{1 5 , 8 0}$

Verglichen mit neueren Versuchen von J. Violle:

$\begin{array}{cc}\text { Temperatur } & \begin{array}{c}\text { Vioile } \\ \text { mit Platin }\end{array} \\ 800 & 0,059 \\ 900 & 0,26 \\ 1000 & 1 \\ 1100 & 3,35 \\ 1300 & 25 \\ 1500 & 107 \\ 1700 & 264 \\ 1775 & 323\end{array}$

\begin{tabular}{cc}
\multicolumn{3}{c}{ Le Chatelier } \\
mit Fisen oxyd & mit Platin \\
0,038 & 0,024 \\
0,26 & 0,27 \\
$\mathbf{1}$ & $\mathbf{1}$ \\
$\mathbf{3}, 1$ & $\mathbf{3 , 2}$ \\
$\mathbf{2 1}$ & 23 \\
$\mathbf{8 6}$ & 98 \\
290 & 340 \\
385 & 450
\end{tabular}

Wärmemessung. Zur Messung hoher Temperaturen verglichen L. Holborn und W. Wien (Z. Instr. 1892 S. 259) das Ther- moelement von Le Chatelier (d. Z. 1891 S. 60) mit dem Luftthermometer. Die mit dem so richtig gestellten Pyrometer bestimmten Schmelzpunkte ergaben:

$\begin{array}{lc}\text { Gold } & 1072^{\circ} \\ \text { Silber } & 968 \\ \text { Kupfer } & 1082\end{array}$

Zur Herstellung hochgradiger Quecksilberthermometer empfiehlt A. Mahlke (Z. Instr. 1892 S. 402) die Verwendung flüssiger Kohlensäure, um den erforderlichen Druck von 17 bis $18 \mathrm{Atm}$. zu erzeugen. Mit Hülfe des Glases $59^{\text {III }}$ von Schott in Jena lassen sich so Quecksilberthermometer erzeugen, welche genaue Temperaturmessungen bis $550^{\circ}$ ermöglichen.

Quecksilberthermometer von Schott \& Gen. (D.R.P. No. 68140). Bei Quecksilberthermometern mit kleinem Gefäss wird die Scaleneintheilung so fein, dass sie nur schwer erkennbar wird. Zur Vermeidung dieses Übelstandes wird nun vor das Thermometerrohr eine vergrössernd wirkende Glaslamelle angeordnet, so dass die durch diese Lamelle betrachtete Scala vergrössert erscheint.

Für Lichtmessungen empfiehlt $N$. Teclu (J. pr. Chem. 47 S. 568) das Radiometer, da seiner Ansicht nach die Geschwindigkeit der Umdrehung des Schaufelrades im graden Verbältniss steht zu den Lichtstärken.

Auslaugen im Vacuum. Um nach C. Heckmann (D.R.P. No. 68980) Farbhölzer, Knochen, Wolle u. dgl. Stoffe, welche wegen ihrer Zellenstructur und der im Innern derselben eingeschlossenen Luft dem Auslaugemittel nur schwer den Eintritt gestatten, in einer verhältnissmässig kurzen Zeit und möglichst vollkommen auslaugen zu können, hat man das Verfahren eingeschlagen, aus diesen Körpern die Luft auszupumpen und dieselben dann mit dem Auslaugemittel in Berührung zu bringen. Wenn aber, wie dies häufig der Fall ist, das Auslaugemittel Gase enthält, welche im luftverdünnten Raum aus der Flüssigkeit entweichen, so werden beim Uebergiessen der auszulaugenden Stoffe mit jener Flüssigkeit die entwickelten Gase in die zuvor luftleer gepumpten Hohlräume des Auslaugegutes eindringen und die Flüssigkeit an einem Eintritt in das letztere verhindern.

Um nun dies zu vermeiden, wird vor der Einführung der gasentwickelnden Löseflüssigkeit in das Auslaugegefäss zunächst ent- 
lufteter Wasserdampf ron ungefähr Atmosphärenspannung in das letztere eingeleitet, so dass er in die zuvor luftleer gepumpten Hohlräume des Auslaugegutes eindringen kann. Erst nachdem dies geschehen ist und während die Masse beständig unter Dampfdruck gehalten wird, leitet man das Lösemittel ein, bis es den auszulaugenden Stoff vollkommen bedeckt. Alsdann kann man das Gefäss mit seinem Inhalt sich abkühlen und $u$. $D$. auch von aussen Luft zutreten lassen. Der in das Auslaugegut eingedrungene Wasserdampf wird hierbei verfüssigt und die dadurch im Innern des auszulaugenden Stoffes gebildeten luftrerdünnten Räume saugen das Auslaugemittel selbstthätig ein, so dass der auszulaugende Stoff von der betreffenden Flüssigkeit vollkommen durchdrungen wird.

\section{Nene Bücher.}

Ferd. Fischer: Taschenbuch für Feuerungstechniker. Kurze Anleitung zur Untersuchung und Beurtheilung von Feuerungsanlagen. 2. Aufl. (Stuttgart, J. G. Cotta'sche Buchb. Nachf.) $96 \mathrm{~S}$.

Vorliegendes, für Chemiker, Hüttenleute, Dampfkesselingenieure, Gasanstalten u.s.w. bestimmte Taschenbuch soll eine kurze Anleitung geben, wie man auch während des Betriebes rasch den Gang des Verbrennungsprocesses und die Ausnutzung der erzeugten Wärme feststellen und darnach, wenn erforderlich, verbessernd eingreifen kann.

E. Dorn: Vorschläge zu gesetzlichen Bestimmungen über elektrische Maasseinheiten, entworfen durch das $\mathrm{Cu}$ ratorium der Physikalisch-Technischen Reichsanstalt. (Berlin, Julius Springer.)

Ausser den Vorschlägen bringt die Schrift einen sehr gründlichen kritischen Bericht über den wahrscheinlichen Werth des $0 \mathrm{hm}$ nach den bisherigen Messungen.

Lothar Meyer: Grundzüge der theoretischen Chemie. 2. Aufl. (Leipzig, Breitk opf \& Härtel.) Pr. 4 M.

Dass für die "Grundzüge" schon nach zwei Jahren eine neue Auflage nōthig war, zeigt schon, welchen Beifall das Buch unter den Fachgenossen mit Recht findet. Es sei bestens empfohlen.

G. Krüss: Zeitschrift für anorganische Chemie. (Hamburg, Leopold Voss.)

Die vorliegenden zwei ersten Bände dieser neuen Zeitschrift bringen zahlreiche Originalarbeiten und kurze Berichte aus anderen Zeitschriften über die sonstigen Arbeiten auf dem Gebiete der (reinen) anorganischen Chemie.
A. Pinner: Die Imidoather und ihre Derivate. (Berlin, R. Oppenheim.) Pr. 7 M. Eine übersichtliche Zusammenfassung der zerstreuten Arbeiten über Imidoäther, welche allen organisch arbeitenden Chemikern willkommen sein wird.

W. Borchers: Anorganische Chemie. (Braunschweig, H. Bruhn.) Pr. 2,80 M.

Die vorliegende Einführung in die Grundlehren der Chemie nebst kurzem Leitfaden zur anorganischchemischen Technologie unter besonderer Berücksichtigung der Metallurgie, ist zum Gebrauche an technischen Fachschulen und Realschulen bestimmt und dafür auch recht gut geeignet.

\section{Patentanmeldnngen.}

Klasse:

\section{(R. A. 4. Mai 1893.)}

6. P. 6047. Verfahren und Apparat zum Destilliren und Rectificiren - G. Plath in Cassel. 17. Dec. 1892.

8. W. 8961. Spulenträger für Färbemaschinen und dergl. - J. Wülfing \& Sohn in Lennep. 21. Febr. 1893

10. R. 7890. Vorrichtung zum Einebnen der Koble in liegenden Koksöfen. - A. Reinecken in Düsseldorf. 27. Febr. 1893 .

22. C. 4374. Verfahren zur Darstellung einer $\alpha_{1} \alpha_{4}-\mathrm{Naph}$ tylendiamindisalfosäure. - Leopold Cassella \& Co. in Frankfurt a. M. 7. Dec. 1892 .

- F. 6445. Verfahren zar Darstellung von Nitrofarbstoffen der Rhodaminrelhe. (Zus. z. P.-Anm. F. 6327.) Farbwerke vorm. Meister Lucius \& Brüning in Höchst a. M. 19. Dec. 1892 .

- L. 7710. Verfahren zur Herstellung von baltbarer Leimgallerte für Holzverbindungen. - E. Lehmann in Oschersleben. 11. Nov. 1892.

- S. 6260. Verfahren zur Darstellung von Bloiweiss aus dem Bleioxyd des Handels. - W. Smith und W. EImore in London. 31. Oct. 1891.

\section{(R. A. 8. Mai 1893.)}

22. B. 13696. Verfahren zur Darstellung benzylirter Ketone ans Tetrametbyl- bez. Tetraäthyldiamidobenzophenon. - Badische Anilin- und Sodafabrik in Ludwigshafen a. Rh. 8. Sept. 1892 .

- B. 14 102. Verfahren zur Darstellung blaner Farbstoffe sauren Characeters aus Nitrosodialkyl-m-amidophenol. (Zus. z. P. No. 45 268.) - Badische Anilin- und Sodafabrik in Ludwigshafen a. Rh. 19. Dec. 1892.

75. C. 4492. Vorbereitung des Salzes für den HargreavesProcess. - Chemische Fabrik Rhenania in Aachen. 9. März 1893.

\section{(R. A. 12. Mai 1893.)}

12. H. 13309. Verfabren zur Darstellung von Vanillin. F. von Heyden Nachf. in Radebeul. 13. Juli 1892.

- Sch. 7996. Verfahren zum Entfärben und Klären von gerbstoffhaltigen Flüssigkeiten. - A. H. Schenk in Hamburg 2. 3. Mai 1892.

18. St. 3421. Vorbereitung des Roheisens für den Frischprocess. - Staffordshire Steel \& Ingot Iron Company in Bilston. 22. Nov. 1892.

22. C. 4479 . Verfahren zur Darstellung von $\alpha_{1}-$ Amido- $\alpha_{3}$ naphtol- $\beta_{1}$-monosulfosĩure. - Chemische Fabrik Grünau - Landshoff \& Meyer - in Grünau bei Berlin. 27. Febr. 1893 .

23. Sch. 8574. Schmelzgefässe für Fett. (Zus. z. Pat. No. 68 829.) - Scheffel \& Shiel in Mülbeim a. Rh. 31. Jan. 1893.

75. S. 6782 . Anode zur Elektrolyse von Salzlösungen. W. Spilker, Berlin. 13. Ang. 1892.

\section{(R. A. 15. Mai 1893.)}

8. E. 3778 . Verfahren zum Färben von Haaren und Federn mit Sulfosäuren des p-Amidophenols und p-Phevy'endiamins. (Zus. z. Pat. No. 47 349.) - H. Erdmann in Halle a. S. 22. März 1893.

10. C. 4442 . Liegender Koksofen. - F. J. Collin in Dortmund. 31. Jan. 1893. 